\title{
Research on Multi-Model of Computer Organization for System Capability
}

\author{
Li HUANG ${ }^{1,2,3, a, *}$, Wei HU $U^{1,2,3, b}$, Kai ZHANG ${ }^{1,2,3, \mathrm{c}}$ and Jianhua LU $\mathrm{U}^{1,2,3, \mathrm{~d}}$ \\ ${ }^{1}$ College of Computer Science and Technology, Wuhan University of Science and Technology, \\ Hubei, Wuhan, China \\ ${ }^{2}$ Hubei Province Key Laboratory of Intelligent Information Processing and Real-time Industrial \\ System, Wuhan University of Science and Technology, Hubei, Wuhan, China \\ ${ }^{3}$ National Computer Experimental Teaching Demonstration Center (Wuhan University of Science \\ and Technology), Hubei, Wuhan, China \\ ahuangli82@wust.edu.cn, bhuwei@wust.edu.cn, ${ }^{\text {c}}$ zhangkai@wust.edu.cn, ${ }^{\mathrm{d}}$ lujianhua@wust.edu.cn, \\ ${ }^{*}$ corresponding author
}

Keywords: system capability, multiple teaching mode, computer organization

\begin{abstract}
This paper studies the lack of systematic, teaching content and the obsolete teaching mode of the principle of computer composition in most colleges and universities in China. Proposed system-oriented teaching methods and modes. This paper expounds the teaching goal of cultivating system competence based on classroom teaching system, supported by gradient experiment teaching, combining MOOC platform and SPOC teaching mode, and taking the second class as the teaching implementation system expanded.
\end{abstract}

\section{Introduction}

With the advent of mobile Internet, important changes have taken place in the form of the information industry. The application of new computer systems is deepening and the training of computer professionals must also keep pace with the times. The training of computer professionals with systematic capabilities has become the consensus of education experts at home and abroad.

The principle of computer composition is the most important core professional basic course in computer science. It focuses on the micro-computer's structural composition, logic functions and internal operation mechanism. The pilot course is a digital logic circuit. It is also the basis of computer system structure, compilation principle, operating system and other follow-up courses. It plays a key role in the teaching of the whole computer specialty. It is an essential part of developing a student's concept of building a computer system. Through this course, students have a complete understanding of the hierarchy of computer systems and a deep understanding of the basic principles of computer work. Making the student clear the relationship between computer software and hardware, and Easier to understand from the perspective of the computer system as a whole operating system, compiler theory and other follow-up course content. Forming a complete concept of computer system concept, and finally have the ability of analysis, application, design and development of the computer system.

\section{Situation Analysis}

The course of Computer Organization is characterized by a wide range of knowledge, large amount of teaching information and rapid updating of teaching content. However, many students feel boring about their studies and have no enthusiasm for taking part in the programs due to the theoreticality of the courses and the absurdity of the basic concepts. Students are only learning passively, they can not realize the fun of learning, and can not cultivate the ability of innovation. The traditional teaching is mainly to impart knowledge. It ignores capacity-building and engineering exercises. The idea of engineering can not be reflected in the course, and the understanding of the project is not enough. In engineering practice, poor interpersonal skills, such as teamwork, communication and 
communication skills have not been effectively cultivated. Students are less trained in team - based projects. And having strong individual consciousness and lack of teamwork spirit. Moreover, the traditional assessment methods mainly focus on the mastery of the theory, appraisal of practice is obviously inadequate. The single method of assessment leads to the incoordination of knowledge and ability training, and the results of the assessment are not fair enough.

Massive open online courses (MOOC)[1] are emerging forms of online courses based on curriculum and pedagogy and networking and mobile intelligence technologies. Many famous teachers provide rich teaching resources for them.

Small private online course (SPOC)[2] teaching mode, which utilizes MOOC technology and resources, combines classroom teaching with online teaching and implements flipped classroom teaching. It has attracted wide attention in foreign universities, and has achieved good teaching results.

Using MOOC resources to better learn from the teaching experience of foreign universities and to solve the problems in the course of computer composition teaching in most colleges and universities in China. It has become the focus of the research and reform of computer hardware teaching in China to meet the requirements of teaching goal of system level design, realization and application ability training.

The teaching reform of hardware and software integration and system oriented design ability will enable students to understand the computer deeply. Students have the ability to use the computer system to solve practical problems and improve the overall quality of the society to meet the needs of the society.

\section{Computer System Ability Training}

For computer system training, teaching reform requires teachers to have their own computer software and hardware systems in-depth understanding, has a strong hands-on ability. It is required that the preorder class cooperate with the following class teaching group, and the teachers of different specialties cooperate with each other. Students also need to spend more time to complete the course practice after school. This puts forward higher requirements on students' self-management, self-study and practical ability.

System Construction Classroom Teaching System. Classroom content can be borrowed from the curricula of top US universities such as Stanford University[3], UC[4], Carnegie Mellon University[5] (CMU),Berkeley . In order to train computer programmers from the perspective of programmers, understand program execution from the perspective of hardware designers, teach the basic working principles of computer hardware, and the correlation between hardware and software. Forming a teaching syllabus that aims to develop the system's ability to lay the foundation for the first level of learning in a hierarchical computer system.

First of all, teaching should focus on mastering the principle of computer hardware, reducing the design details of components. With the design function module as the precursor, the single cycle CPU is the transition, and the pipeline CPU is finally realized. For the important knowledge points, such as instruction system design, CPU model machine, data access, Cache mechanism, etc., the transformation mechanism from single cycle CPU to pipelined CPU should be established to embody the new technology and new concept in computer field. For example, contrasting the similarities and differences and advantages and disadvantages of CPU based on RISC or CISC instruction set in design, instruction register, bus path, reading mechanism, time series control and so on.

Secondly, the support of the upper program is supported by the computer composition and working principle, and the connection between the hardware design and the software is emphasized. For example, information representation and data definition of $\mathrm{C}$ language; machine level instruction and $\mathrm{C}$ program's machine level representation; data path and program execution; memory structure and $C$ program's scheduling and execution; processor architecture and program execution efficiency. At the same time, we build a typical set of $C$ language programming cases and disassemble them, and describe how the computer interpreter the execution program in principle. 
What parts of the system will affect the execution efficiency of the program, and how to avoid or reduce the impact. Helping students to improve the performance of the program based on the understanding of the computer system and construct a hierarchical computer system.

Finally, we should integrate new technologies and concepts of computers appropriately, such as parallel computers, distributed computers, and cloud service architectures, etc.. Improve the students' interest in the curriculum and carry out innovative research.

Gradient Experiment Teaching System. For most students, a confirmatory experiment on a highly integrated computer module is difficult to understand the nature of the experimental phenomena, except for wiring and experimental conclusions. Therefore, to construct a set of gradient experimental teaching system that accords with classroom teaching system is conducive to the internalization of system competence.

First of all, the construction of basic experimental cases. Design experiments to understand the design principles of each functional component of a computer, such as ALU experiment, micro program control experiment, memory experiment, I/O experiment, etc. Students are required to use Proteus and other software to simulate the design to deepen the understanding of the functional components. From the perspective of computer supported upper layer program, we designed experiments, such as data definition experiment, binary bomb, architecture experiment, buffer overflow, performance test and so on. So students can explain the various phenomena of program writing based on the understanding of the working principle of the computer, so as to make better programming.

Secondly, design the data path and timing system based on the design of the functional components as the leading and single-cycle work. Using Proteus software to simulate the computer to fetch instructions and execute the complete process of instruction to design of single-cycle CPU system. Finally, the single cycle CPU is optimized and improved, and the pipelined CPU is finally designed. Students can design different series of pipelines based on their own abilities. Teachers select highly competent, passionate, challenged and innovative students and their work to further optimize the performance of the CPU core and moderately increase other functions such as forwarding and blocking.

\section{Diversified Teaching Methods and Organization}

Construction of Three-dimensional Curriculum Resources. With the classroom and experimental teaching system, each chapter will be broken down into a number of relatively independent knowledge points, elaborate course notes and courseware, planning course video. Video construction adopts a step-by-step strategy. At the beginning of the teaching reform, MOOC video with moderate difficulty was taken as the main body. The rest of the related MOOC videos were supplemented. Through the selection and arrangement, a video system and implementation plan suitable for our school was formed. With the accumulation of experience and teaching needs, perfect curriculum video will be made after taking full consideration of the matching relationship between the course and other courses and experimental teaching.

Extensive use of MOOC resources, the construction of online exam system planning, discuss problem sets, the process of monitoring and evaluation system; tracking, collection and computer hardware technology development of new theories and technologies related to the data and references, let students put their spare time, according to the study and research points of interest, the classroom after class learning and expand organically, stimulate learning interest and enthusiasm.

SPOC Teaching Mode. Organize all the teaching resources organically, set up an online course platform and implement the SPOC teaching mode. The students are divided into groups, all classroom teaching and online teaching are carried out in groups as a unit. The teachers arrange the online learning video, the relevant literature, the homework, the learning requirements, the completion time and the classroom discussion. Students are divided into groups to study and discuss, to refine the knowledge framework, difficult and difficult, and the existing problems, to write the study research report, and to prepare for the full report. In class, each group completed the specified 
number of class reports, other classmates participated in the class discussion, and students who did not have the opportunity to speak could continue to participate in the discussion online. Teachers in the classroom review of knowledge, concatenation, development and deepening, to answer students' questions, work with students or other tasks to ensure teaching effectiveness. And regularly publish tests, online discussions, etc. to get feedback information on the effect of learning.

Course Evaluation System Based on Process Assessment. We should explore the tracking mechanism of learning effect, integrate online learning, classroom learning and experimental learning scoring mechanism to form a comprehensive evaluation mechanism that runs through the whole curriculum, and fundamentally change the unscientific evaluation mode. For example: the use of process monitoring and evaluation system, online learning, students' scores from video work, mutual evaluation work, test, online discussions grade comprehensive; classroom performance by the group to participate in the new report, technical questions and discussion, class (students), class for industry comprehensive achievement; in view of the experimental teaching. Exploration team scoring mechanism, experimental innovation mechanism, plus comprehensive curriculum design mechanism, in practice from multi angle test students.

Combined with Modern Teaching Methods. In order to mobilize the students' interest in learning, deepen their understanding and inspire their thinking, teachers should widely adopt various teaching methods and means to ensure the system's ability to be cultivated. The analogy teaching method when explaining the abstract principle, derived from real life or ideological affairs into the classroom, help students understand, to abstract problems, such as: the line is similar to the instruction pre hospital queue treatment, the working principle of interrupt is similar to the students in the classroom questioning, the principle is similar to the extended memory the classroom seating arrangement etc. At the same time, the use of animation image teaching, for example, the working principle of CPU line between a computer module and module with each other to complete, if the player PPT page teaching method is obviously difficult to form a conceptual system; can use Author ware, Flash animation, VBA programming and other modern means of Education, knowledge of the integration by teachers, by way of animation intuitively show the whole process of data processing and transmission, so the negative impact can to some extent avoid the hardware abstraction theory brings to the teaching.

Forming Distinctive Second Class. With cloud computing, mobile Internet and other frontier combination in the professional range, widely carry out the principle and form of ability and training of computer second class related activities, such as the development of CPU creative design contest, exchange student entries in the water treatment system, Cache mapping mechanism, conflict handling, forwarding, blocking treatment and other aspects performance. Customizing the computer design competition to allow students to consider, tailor and optimize the design of CPU, from the cost-effective and customizable performance. CPU secondary development competition, to encourage students and computer science frontier, in their own design of the CPU for game development, robotics design, Internet of Things applications, mobile Internet applications, to stimulate student innovation. Outstanding works in the competition stand out, teachers can guide students to further optimize and improve, so that students on a broader platform for exchange, learning, access to a more depth and breadth of system skills training, and then to reach the pre-match, race Promote a virtuous circle of learning.

\section{Summary}

Although the principle of computer organization is a curriculum emphasizing on hardware, it is the foundation of many systems and software courses, and its practicality is very strong. It requires students to fully understand and master the working principles and specific structures of computers in the process of practice. The new era of computer professionals need to master the basic working principle of computer systems and understand the interaction between computer hardware and software systems, with system design and system application capabilities. Curriculum reform oriented to system competence needs to be constantly perfected in the process of implementation. We should optimize teaching design, show the new teaching idea with advanced theory, and further 
explore the new educational field of 'solid theoretical basis and innovative skills' in the teaching of computer hardware, and stimulate students' creative thinking.

\section{Acknowledgment}

In this paper, the research was sponsored by the Ministry of education cooperation in collaborative education ( No. 201702134010) and Hubei Province teaching and research ( No. 2016237).

\section{References}

[1] Lee G. Professor William Fisher's edX, CopyrightX MOOC[EB/OL]. [2013-05-09]. http: //masslawblog. com/copyright/observations-on-professor-william-fi shers-edx-copyrightx-mooc/

[2] The joint task force on computing curricula of ACMIIEEE. ComputerScience Curricula 2013 Iromnan Draft (Version 0.8)[EB/OL]. [2013-03-26]. http: //ai. stanford. edu/users/sahami/CS2013/

[3] Standford University. Computer science courses[EB/OL]. [2013-07-02]. http: //www. cs.stanford. edu/courses

[4] UC Berkeley. CS courses information \& scheduling[EB/OL]. [2013-07-02]. http: //www. eecs. berkeley. edu/Scheduling/CS/

[5] Caron P. Harvard Law School offers first free online course[EB/OL]. [2012-12-28]. http: //taxprof. typepad. com/taxprof_blog/2012/12/harvard-law-school. html 